\title{
Strong convergence of a general iterative algorithm in Hilbert spaces
}

Songtao LV*

"Correspondence: sqlvst@yeah.net School of Mathematics and Information Science, Shangqiu Normal University, Shangqiu, 476000, China

\begin{abstract}
In this paper, the problem of approximating a common element in the common fixed point set of an infinite family of nonexpansive mappings, in the solution set of a variational inequality involving an inverse-strongly monotone mapping and in the solution set of an equilibrium problem is investigated based on a general iterative algorithm. Strong convergence of the iterative algorithm is obtained in the framework of Hilbert spaces. The results obtained in this paper improve the corresponding results announced by many authors.
\end{abstract}

AMS Subject Classification: 47H09; 47J05; 47J25

Keywords: nonexpansive mapping; viscosity approximation method; equilibrium problem; fixed point

\section{Introduction and preliminaries}

Let $H$ be a real Hilbert space, whose inner product and norm are denoted by $\langle\cdot, \cdot\rangle$ and $\|\cdot\|$ respectively. Let $C$ be a nonempty, closed and convex subset of $H$ and $T: C \rightarrow C$ be a mapping. In this paper, we use $F(T)$ to denote the set of fixed points of $T$. Recall that $T$ is said to be a $\kappa$-contraction iff there exists a constant $\kappa \in(0,1)$ such that

$$
\|T x-T y\| \leq \kappa\|x-y\|, \quad \forall x, y \in C
$$

$T$ is said to be a nonexpansive mapping iff

$$
\|T x-T y\| \leq\|x-y\|, \quad \forall x, y \in C .
$$

Let $B: C \rightarrow H$ be a mapping. Recall that $B$ is said to be an $\alpha$-inverse-strongly monotone iff there exits a positive constant $\alpha$ such that

$$
\langle B x-B y, x-y\rangle \geq \alpha\|B x-B y\|^{2}, \quad \forall x, y \in C .
$$

The classical variational inequality is to find $u \in C$ such that

$$
\langle B u, v-u\rangle \geq 0, \quad \forall v \in C .
$$

In this paper, we use $V I(C, B)$ to denote the solution set of the variational inequality. 
Let $P_{C}$ be the metric projection from $H$ onto $C$. It is also known that $P_{C}$ satisfies

$$
\left\langle x-y, P_{C} x-P_{C} y\right\rangle \geq\left\|P_{C} x-P_{C} y\right\|^{2}, \quad \forall x, y \in H .
$$

Moreover, $P_{C} x$ is characterized by the properties $P_{C} x \in C$ and $\left\langle x-P_{C} x, P_{C} x-y\right\rangle \geq 0$ for all $y \in C$. One can see that the variational inequality is equivalent to a fixed point problem. The element $u \in C$ is a solution of the variational inequality if and only if $u$ is a fixed point of the mapping $P_{C}(I-\lambda B)$, where $\lambda>0$ is a constant and $I$ is the identity mapping. This alternative equivalent formulation has played a significant role in the studies of the variational inequality and related optimization problems.

Recall that an operator $A$ is strongly positive on $H$ iff there exists a constant $\bar{\gamma}>0$ with the property

$$
\langle A x, x\rangle \geq \bar{\gamma}\|x\|^{2}, \quad \forall x \in H .
$$

Recall that a set-valued mapping $S: H \rightarrow 2^{H}$ is said to be monotone if for all $x, y \in H$, $f \in S x$ and $g \in S y$ imply $\langle x-y, f-g\rangle \geq 0$. A monotone mapping $S: H \rightarrow 2^{H}$ is maximal if the graph of $\operatorname{Graph}(S)$ of $S$ is not properly contained in the graph of any other monotone mapping. It is known that a monotone mapping $S$ is maximal iff for $(x, f) \in H \times H,\langle x-$ $y, f-g\rangle \geq 0$ for every $(y, g) \in \operatorname{Graph}(S)$ implies $f \in S x$. Let $B$ be a monotone map of $C$ into $H$ and let $N_{C} v$ be the normal cone to $C$ at $v \in C$, i.e., $N_{C} v=\{w \in H:\langle v-u, w\rangle \geq 0, \forall u \in C\}$ and define

$$
S v= \begin{cases}B v+N_{C} v, & v \in C, \\ \emptyset, & v \notin C .\end{cases}
$$

Then $S$ is maximal monotone and $0 \in S v$ iff $v \in V I(C, B)$; see [1] and the references therein.

Let $F$ be a bifunction of $C \times C$ into $\mathbb{R}$, where $\mathbb{R}$ is the set of real numbers. The equilibrium problem for $F: C \times C \rightarrow \mathbb{R}$ is to find $x \in C$ such that

$$
F(x, y) \geq 0, \quad \forall y \in C .
$$

The set of solutions of the problem (1.2) is denoted by $E P(F)$. Numerous problems in physics, optimization and economics reduce to finding a solution of (1.2). Recently, many iterative algorithms have been studied to solve the equilibrium problem (1.2); see, for instance, [2-19].

For solving the equilibrium problem (1.2), let us assume that $F$ satisfies the following conditions:

(A1) $F(x, x)=0$ for all $x \in C$;

(A2) $F$ is monotone, i.e., $F(x, y)+F(y, x) \leq 0$ for all $x, y \in C$;

(A3) for each $x, y, z \in C$,

$$
\limsup _{t \downarrow 0} F(t z+(1-t) x, y) \leq F(x, y)
$$

(A4) for each $x \in C, y \mapsto F(x, y)$ is convex and lower semicontinuous.

In 2007, Takahashi and Takahashi [17] proved the following result. 
Theorem TT Let $C$ be a nonempty closed convex subset of $H$. Let $F$ be a bifunction from $C \times C$ to $R$ satisfying (A1)-(A4) and let $T$ be a nonexpansive mapping of $C$ into $H$ such that $F(S) \cap E P(F) \neq \emptyset$. Let $f$ be a contraction of $H$ into itself and let $\left\{x_{n}\right\}$ and $\left\{u_{n}\right\}$ be sequences generated by $x_{1} \in H$ and

$$
\left\{\begin{array}{l}
F\left(y_{n}, u\right)+\frac{1}{r_{n}}\left\langle u-y_{n}, y_{n}-x_{n}\right\rangle \geq 0, \quad \forall u \in C, \\
x_{n+1}=\alpha_{n} f\left(x_{n}\right)+\left(1-\alpha_{n}\right) T y_{n}, \quad n \geq 0,
\end{array}\right.
$$

where $\left\{\alpha_{n}\right\} \subset[0,1]$ and $\left\{r_{n}\right\} \subset(0, \infty)$ satisfy $\lim _{n \rightarrow \infty} \alpha_{n}=0, \sum_{n=1}^{\infty} \alpha_{n}=\infty, \sum_{n=1}^{\infty} \mid \alpha_{n+1}-$ $\alpha_{n}\left|<\infty, \sum_{n=1}^{\infty}\right| r_{n+1}-r_{n} \mid<\infty$, and $\liminf _{n \rightarrow \infty} r_{n}>0$. Then $\left\{x_{n}\right\}$ and $\left\{y_{n}\right\}$ strongly converge to some point $z$, where $z=P_{C} F(T) \cap E P(T) f(z)$.

Recently, Plubtieng and Punpaeng [19] further improved the above results by involving a strongly positive self-adjoint operator. To be more precise, they proved the following results.

Theorem PP Let $H$ bea real Hilbertspace, let $F$ be a bifunction from $H \times H \rightarrow R$ satisfying (A1)-(A4) and let $T$ be a nonexpansive mapping on $H$ such that $F(T) \cap E P(F) \neq \emptyset$. Let $f$ be a contraction of $H$ into itself with $\alpha \in(0,1)$ and let $A$ be a strongly positive bounded linear operator on $H$ with the coefficient $\bar{\gamma}>0$ and $0<\gamma<\frac{\bar{\gamma}}{\alpha}$. Let $\left\{x_{n}\right\}$ be a sequence generated by $x_{1} \in H$ and

$$
\left\{\begin{array}{l}
F\left(y_{n}, u\right)+\frac{1}{r_{n}}\left\langle u-y_{n}, y_{n}-x_{n}\right\rangle \geq 0, \quad \forall u \in C, \\
x_{n+1}=\alpha_{n} \gamma f\left(x_{n}\right)+\left(I-\alpha_{n} A\right) T y_{n}, \quad n \geq 1,
\end{array}\right.
$$

where $\left\{\alpha_{n}\right\} \subset[0,1]$ and $\left\{r_{n}\right\} \subset(0, \infty)$ satisfy $\lim _{n \rightarrow \infty} \alpha_{n}=0, \sum_{n=1}^{\infty} \alpha_{n}=\infty, \sum_{n=1}^{\infty} \mid \alpha_{n+1}-$ $\alpha_{n}\left|<\infty, \sum_{n=1}^{\infty}\right| r_{n+1}-r_{n} \mid<\infty$, and $\liminf _{n \rightarrow \infty} r_{n}>0$. Then $\left\{x_{n}\right\}$ and $\left\{y_{n}\right\}$ strongly converge to some point $z$, where $z=P_{F(T) \cap E P(T)}(I-A+\gamma f)(z)$.

In 2008, Su, Shang and Qin [2] considered the variational inequality (1.1), and the equilibrium problem (1.2) based on a composite iterative algorithm and proved the following theorem.

Theorem SSQ Let $C$ be a nonempty closed convex subset of $H$. Let $F$ be a bifunction from $C \times C$ to $R$ satisfying (A1)-(A4). Let $A$ be $\alpha$-inverse-strongly monotone and let $T$ be a nonexpansive mapping of $C$ into $H$ such that $F(S) \cap E P(F) \cap V I(C, A) \neq \emptyset$. Let $f$ be a contraction of $H$ into itself and let $\left\{x_{n}\right\}$ and $\left\{u_{n}\right\}$ be sequences generated by $x_{1} \in H$ and

$$
\left\{\begin{array}{l}
F\left(y_{n}, u\right)+\frac{1}{r_{n}}\left\langle u-y_{n}, y_{n}-x_{n}\right\rangle \geq 0, \quad \forall u \in C, \\
x_{n+1}=\alpha_{n} f\left(x_{n}\right)+\left(1-\alpha_{n}\right) T P_{C}\left(I-\lambda_{n} A\right) y_{n}, \quad n \geq 0,
\end{array}\right.
$$

where $\left\{\lambda_{n}\right\} \subset[a, b]$, where $0<a<b<2 \alpha,\left\{\alpha_{n}\right\} \subset[0,1]$ and $\left\{r_{n}\right\} \subset(0, \infty)$ satisfy $\lim _{n \rightarrow \infty} \alpha_{n}=$ $0, \sum_{n=1}^{\infty} \alpha_{n}=\infty, \sum_{n=1}^{\infty}\left|\alpha_{n+1}-\alpha_{n}\right|<\infty, \sum_{n=1}^{\infty}\left|r_{n+1}-r_{n}\right|<\infty, \sum_{n=1}^{\infty}\left|\lambda_{n+1}-\lambda_{n}\right|<\infty$, and $\liminf _{n \rightarrow \infty} r_{n}>0$. Then $\left\{x_{n}\right\}$ and $\left\{y_{n}\right\}$ strongly converge to some point $z$, where $z=$ $P_{C} F(T) \cap E P(T) f(z)$. 
The above results only involve a single mapping, we will consider an infinite family of mappings in this paper. To be more precise, we study the mapping $W_{n}$ defined by

$$
\begin{aligned}
& U_{n, n+1}=I, \\
& U_{n, n}=\gamma_{n} T_{n} U_{n, n+1}+\left(1-\gamma_{n}\right) I, \\
& U_{n, n-1}=\gamma_{n-1} T_{n-1} U_{n, n}+\left(1-\gamma_{n-1}\right) I, \\
& \vdots \\
& U_{n, k}=\gamma_{k} T_{k} U_{n, k+1}+\left(1-\gamma_{k}\right) I, \\
& u_{n, k-1}=\gamma_{k-1} T_{k-1} U_{n, k}+\left(1-\gamma_{k-1}\right) I, \\
& \vdots \\
& U_{n, 2}=\gamma_{2} T_{2} U_{u, 3}+\left(1-\gamma_{2}\right) I, \\
& W_{n}=U_{n, 1}=\gamma_{1} T_{1} U_{n, 2}+\left(1-\gamma_{1}\right) I,
\end{aligned}
$$

where $\left\{\gamma_{1}\right\},\left\{\gamma_{2}\right\}, \ldots$ are real numbers such that $0 \leq \gamma_{n} \leq 1, T_{1}, T_{2}, \ldots$ are an infinite family of mappings of $C$ into itself.

Considering $W_{n}$, we have the following lemmas which are important in proving our main results.

Lemma 1.1 [20] Let $C$ be a nonempty closed convex subset of a strictly convex Banach space E. Let $T_{1}, T_{2}, \ldots$ be nonexpansive mappings of $C$ into itself such that $\bigcap_{n=1}^{\infty} F\left(T_{n}\right)$ is nonempty, and let $\gamma_{1}, \gamma_{2}, \ldots$ be real numbers such that $0<\gamma_{n} \leq b<1$ for any $n \geq 1$. Then, for every $x \in C$ and $k \in N$, the limit $\lim _{n \rightarrow \infty} U_{n, k} x$ exists.

Using Lemma 1.1, one can define the mapping $W$ of $C$ into itself as follows:

$$
W x=\lim _{n \rightarrow \infty} W_{n} x=\lim _{n \rightarrow \infty} U_{n, 1} x, \quad \forall x \in C .
$$

Such a $W$ is called the $W$-mapping generated by $T_{1}, T_{2}, \ldots$ and $\gamma_{1}, \gamma_{2}, \ldots$. Throughout this paper, we will assume that $0<\gamma_{n} \leq b<1$, where $b$ is some constant.

Lemma 1.2 [20] Let $C$ be a nonempty closed convex subset of a strictly convex Banach space E. Let $T_{1}, T_{2}, \ldots$ be nonexpansive mappings of $C$ into itself such that $\bigcap_{n=1}^{\infty} F\left(T_{n}\right)$ is nonempty, and let $\gamma_{1}, \gamma_{2}, \ldots$ be real numbers such that $0<\gamma_{n} \leq b<1$ for any $n \geq 1$. Then $F(W)=\bigcap_{n=1}^{\infty} F\left(T_{n}\right)$.

In this paper, based on a general iterative algorithm, we study the problem of approximating a common element in the common fixed point set of an infinite family of nonexpansive mappings, in the solution set of a variational inequality involving an inversestrongly monotone mapping and in the solution set of an equilibrium problem. Strong convergence of the iterative algorithm is obtained in the framework of Hilbert spaces.

In order to obtain the strong convergence, we need the following tools. 
Lemma 1.3 In Hilbert spaces, the following inequality holds:

$$
\|x+y\|^{2} \leq\|x\|^{2}+2\langle y, x+y\rangle, \quad \forall x, y \in H .
$$

Lemma 1.4 [21] Assume that $\left\{\alpha_{n}\right\}$ is a sequence of nonnegative real numbers such that

$$
\alpha_{n+1} \leq\left(1-\gamma_{n}\right) \alpha_{n}+\delta_{n}
$$

where $\left\{\gamma_{n}\right\}$ is a sequence in $(0,1)$ and $\left\{\delta_{n}\right\}$ is a sequence such that

(i) $\sum_{n=1}^{\infty} \gamma_{n}=\infty$;

(ii) $\lim \sup _{n \rightarrow \infty} \delta_{n} / \gamma_{n} \leq 0$ or $\sum_{n=1}^{\infty}\left|\delta_{n}\right|<\infty$.

Then $\lim _{n \rightarrow \infty} \alpha_{n}=0$.

Lemma 1.5 [22] Assume $B$ is a strong positive linear bounded operator on a Hilbert space $H$ with the coefficient $\bar{\gamma}>0$ and $0<\rho \leq\|B\|^{-1}$. Then $\|I-\rho B\| \leq 1-\rho \bar{\gamma}$.

Lemma 1.6 [22] Let $H$ be a Hilbert space. Let $B$ be a strongly positive linear bounded self-adjoint operator with the constant $\bar{\gamma}>0$ and $f$ be a contraction with the constant $\kappa$. Assume that $0<\gamma<\bar{\gamma} / \kappa$. Let $T$ be a nonexpansive mapping with a fixed point $x_{t} \in H$ of the contraction $x \mapsto t \gamma f(x)+(I-t B) T x$. Then $\left\{x_{t}\right\}$ converges strongly as $t \rightarrow 0$ to a fixed point $\bar{x}$ of $T$, which solves the variational inequality

$$
\langle(A-\gamma f) \bar{x}, z-\bar{x}\rangle \leq 0, \quad \forall z \in F(T) .
$$

Equivalently, we have $P_{F(T)}(I-A+\gamma f) \bar{x}=\bar{x}$.

Lemma $1.7[23,24]$ Let $C$ be a nonempty closed convex subset of $H$ and let $B$ be a bifunction of $C \times C$ into $\mathbb{R}$ satisfying (A1)-(A4). Let $r>0$ and $x \in H$. Then there exists $z \in C$ such that

$$
F(z, y)+\frac{1}{r}\langle y-z, z-x\rangle \geq 0, \quad \forall y \in C .
$$

Define a mapping $T_{r}: H \rightarrow C$ as follows:

$$
T_{r}(x)=\left\{z \in C: F(z, y)+\frac{1}{r}\langle y-z, z-x\rangle \geq 0, \forall y \in C\right\} .
$$

Then the following hold:

(1) $T_{r}$ is single-valued;

(2) $T_{r}$ is firmly nonexpansive, i.e., for any $x, y \in H$,

$$
\left\|T_{r} x-T_{r} y\right\|^{2} \leq\left\langle T_{r} x-T_{r} y, x-y\right\rangle
$$

(3) $F\left(T_{r}\right)=E P(F)$;

(4) $E P(F)$ is closed and convex.

Lemma 1.8 [25] Let $\left\{x_{n}\right\}$ and $\left\{y_{n}\right\}$ be bounded sequences in a Banach space $X$ and let $\beta_{n}$ be a sequence in $[0,1]$ with $0<\liminf _{n \rightarrow \infty} \beta_{n} \leq \limsup _{n \rightarrow \infty} \beta_{n}<1$. Suppose $x_{n+1}=\left(1-\beta_{n}\right) y_{n}+$ 
$\beta_{n} x_{n}$ for all integers $n \geq 0$ and

$$
\limsup _{n \rightarrow \infty}\left(\left\|y_{n+1}-y_{n}\right\|-\left\|x_{n+1}-x_{n}\right\|\right) \leq 0 .
$$

Then $\lim _{n \rightarrow \infty}\left\|y_{n}-x_{n}\right\|=0$.

Lemma 1.9 [26, 27] Let $K$ be a nonempty closed convex subset of a Hilbert space $H,\left\{T_{i}\right.$ : $C \rightarrow C\}$ be a family of infinitely nonexpansive mappings with $\bigcap_{i=1}^{\infty} F\left(T_{i}\right),\left\{\gamma_{n}\right\}$ be a real sequence such that $0<\gamma_{n} \leq b<1$ for each $n \geq 1$. If $C$ is any bounded subset of $K$, then $\lim _{n \rightarrow \infty} \sup _{x \in C}\left\|W x-W_{n} x\right\|=0$.

\section{Main results}

Theorem 2.1 Let $C$ be a nonempty closed convex subset of a Hilbert space H. Let $F$ be a bifunction from $C \times C$ to $\mathbb{R}$ which satisfies (A1)-(A4). Let $\left\{T_{n}\right\}_{n=1}^{\infty}$ be an infinite family of nonexpansive mappings of $C$ into $C$. Let $B: C \rightarrow H$ be an $\alpha$-inverse-strongly monotone mapping. Let $A$ be a strongly positive linear bounded self-adjoint operator on $H$ with the coefficient $\bar{\gamma}>0$. Assume that $0<\gamma<\bar{\gamma} / \kappa$ and $F:=\bigcap_{i=1}^{\infty} F\left(T_{i}\right) \cap E P(F) \cap V I(C, B) \neq \emptyset$. Let $f: C \rightarrow H$ be a $\kappa$-contraction. Let $\left\{x_{n}\right\}$ be a sequence generated in the following iterative process:

$$
\left\{\begin{array}{l}
x_{1} \in H, \\
F\left(y_{n}, z\right)+\frac{1}{r_{n}}\left\langle z-y_{n}, y_{n}-x_{n}\right\rangle \geq 0, \quad \forall z \in C, \\
x_{n+1}=\alpha_{n} x_{n}+\left(1-\alpha_{n}\right) \beta_{n} \gamma f\left(y_{n}\right)+\left(1-\alpha_{n}\right)\left(I-\beta_{n} A\right) W_{n} P_{C}\left(I-s_{n} B\right) y_{n}, \quad n \geq 1,
\end{array}\right.
$$

where $W_{n}$ is generated in (1.3), $\left\{\alpha_{n}\right\},\left\{\beta_{n}\right\}$ are real number sequences in $(0,1),\left\{r_{n}\right\}$ and $\left\{s_{n}\right\}$ are positive real number sequences. Assume that the following restrictions are satisfied:

(a) $0<\liminf _{n \rightarrow \infty} \alpha_{n} \leq \limsup _{n \rightarrow \infty} \alpha_{n}<1$;

(b) $\lim _{n \rightarrow \infty} \beta_{n}=0, \sum_{n=1}^{\infty} \beta_{n}=\infty$;

(c) $\lim _{n \rightarrow \infty}\left|r_{n+1}-r_{n}\right|=0, \lim _{n \rightarrow \infty}\left|s_{n+1}-s_{n}\right|=0$;

(d) $\liminf _{n \rightarrow \infty} r_{n}>0,\left\{s_{n}\right\} \subset\left[s, s^{\prime}\right]$ for some $s, s^{\prime}$ with $0<s<s^{\prime}<2 \alpha$.

Then $\left\{x_{n}\right\}$ converges strongly to $q \in F$, where $q=P_{F}(\gamma f+(I-A))(q)$, which solves the following variational inequality:

$$
\langle\gamma f(q)-A q, p-q\rangle \leq 0, \quad \forall p \in F .
$$

Proof We divide the proof into five steps.

Step 1 . Show that the sequence $\left\{x_{n}\right\}$ is bounded.

Notice that $I-s_{n} B$ is nonexpansive. Indeed, we see from the restriction (d) that

$$
\begin{aligned}
& \left\|\left(I-s_{n} B\right) x-\left(I-s_{n} B\right) y\right\|^{2} \\
& \quad=\left\|(x-y)-s_{n}(B x-B y)\right\|^{2} \\
& \quad=\|x-y\|^{2}-2 s_{n}\langle x-y, B x-B y\rangle+s_{n}^{2}\|B x-B y\|^{2} \\
& \quad \leq\|x-y\|^{2}-s_{n}\left(2 \alpha-s_{n}\right)\|B x-B y\|^{2} \\
& \quad \leq\|x-y\|^{2}
\end{aligned}
$$


which implies the mapping $I-s_{n} B$ is nonexpansive. Fix $p \in F$. Since $z_{n}=T_{r_{n}} x_{n}$, we have

$$
\left\|y_{n}-p\right\|=\left\|T_{r_{n}} x_{n}-T_{r_{n}} p\right\| \leq\left\|x_{n}-p\right\| .
$$

Put

$$
\zeta_{n}=\beta_{n} \gamma f\left(y_{n}\right)+\left(I-\beta_{n} A\right) W_{n} \rho_{n},
$$

where

$$
\rho_{n}=P_{C}\left(I-s_{n} B\right) y_{n} .
$$

It follows that

$$
\left\|\rho_{n}-p\right\| \leq\left\|y_{n}-p\right\| \leq\left\|x_{n}-p\right\| .
$$

Since $\beta_{n} \rightarrow 0$ as $n \rightarrow \infty$, we may assume, with no loss of generality, that $\beta_{n}<\|A\|^{-1}$ for all $n$. It follows that

$$
\begin{aligned}
\left\|\zeta_{n}-p\right\| & =\left\|\beta_{n}\left(\gamma f\left(y_{n}\right)-A p\right)+\left(I-\beta_{n} A\right)\left(W_{n} \rho_{n}-p\right)\right\| \\
& \leq \beta_{n}\left\|\gamma f\left(y_{n}\right)-A p\right\|+\left\|I-\beta_{n} A\right\|\left\|W_{n} \rho_{n}-p\right\| \\
& \leq \beta_{n}\left[\gamma\left\|f\left(y_{n}\right)-f(p)\right\|+\|\gamma f(p)-A p\|\right]+\left(1-\beta_{n} \bar{\gamma}\right)\left\|\rho_{n}-p\right\| \\
& \leq \beta_{n}\left[\gamma\left\|f\left(y_{n}\right)-f(p)\right\|+\|\gamma f(p)-A p\|\right]+\left(1-\beta_{n} \bar{\gamma}\right)\left\|x_{n}-p\right\| \\
& \leq\left[1-(\bar{\gamma}-\gamma \kappa) \beta_{n}\right]\left\|x_{n}-p\right\|+\beta_{n}\|\gamma f(p)-A p\|,
\end{aligned}
$$

which yields

$$
\begin{aligned}
\left\|x_{n+1}-p\right\|= & \left\|\alpha_{n}\left(x_{n}-p\right)+\left(1-\alpha_{n}\right)\left(\zeta_{n}-p\right)\right\| \\
\leq & \alpha_{n}\left\|x_{n}-p\right\|+\left(1-\alpha_{n}\right)\left\|\zeta_{n}-p\right\| \\
\leq & \alpha_{n}\left\|x_{n}-p\right\|+\left(1-\alpha_{n}\right)\left[1-(\bar{\gamma}-\gamma \alpha) \beta_{n}\right]\left\|x_{n}-p\right\| \\
& +\left(1-\alpha_{n}\right) \beta_{n}\|\gamma f(p)-A p\| .
\end{aligned}
$$

This in turn implies that

$$
\left\|x_{n}-p\right\| \leq \max \left\{\left\|x_{1}-p\right\|, \frac{\|\gamma f(p)-A p\|}{\bar{\gamma}-\gamma \kappa}\right\} .
$$

This completes the proof that the sequence $\left\{x_{n}\right\}$ is bounded. This completes the proof of Step 1.

Step 2. Show that $\lim _{n \rightarrow \infty}\left\|x_{n+1}-x_{n}\right\|=0$.

In view of $y_{n}=T_{r_{n}} x_{n}$ and $y_{n+1}=T_{r_{n+1}} x_{n+1}$, we see that

$$
F\left(y_{n}, z\right)+\frac{1}{r_{n}}\left\langle z-y_{n}, y_{n}-x_{n}\right\rangle \geq 0, \quad \forall z \in C,
$$


and

$$
F\left(y_{n+1}, z\right)+\frac{1}{r_{n+1}}\left\langle z-y_{n+1}, y_{n+1}-x_{n+1}\right\rangle \geq 0, \quad \forall z \in C .
$$

Putting $z=y_{n+1}$ in (2.1) and $z=y_{n}$ in (2.2), we find that

$$
F\left(y_{n}, y_{n+1}\right)+\frac{1}{r_{n}}\left\langle y_{n+1}-y_{n}, y_{n}-x_{n}\right\rangle \geq 0
$$

and

$$
F\left(y_{n+1}, y_{n}\right)+\frac{1}{r_{n+1}}\left\langle y_{n}-y_{n+1}, y_{n+1}-x_{n+1}\right\rangle \geq 0 \text {. }
$$

It follows from (A2) that

$$
\left\langle y_{n+1}-y_{n}, \frac{y_{n}-x_{n}}{r_{n}}-\frac{y_{n+1}-x_{n+1}}{r_{n+1}}\right\rangle \geq 0 \text {. }
$$

That is,

$$
\left\langle y_{n+1}-y_{n}, y_{n}-y_{n+1}+y_{n+1}-x_{n}-\frac{r_{n}}{r_{n+1}}\left(y_{n+1}-x_{n+1}\right)\right\rangle \geq 0 \text {. }
$$

Without loss of generality, let us assume that there exists a real number $m$ such that $r_{n}>$ $m>0$ for all $n$. It follows that

$$
\left\|y_{n+1}-y_{n}\right\|^{2} \leq\left\|y_{n+1}-y_{n}\right\|\left(\left\|x_{n+1}-x_{n}\right\|+\left|1-\frac{r_{n}}{r_{n+1}}\right|\left\|y_{n+1}-x_{n+1}\right\|\right) .
$$

It follows that

$$
\begin{aligned}
\left\|y_{n+1}-y_{n}\right\| & \leq\left\|x_{n+1}-x_{n}\right\|+\left|1-\frac{r_{n}}{r_{n+1}}\right|\left\|y_{n+1}-x_{n+1}\right\| \\
& \leq\left\|x_{n+1}-x_{n}\right\|+\frac{M_{1}}{m}\left|r_{n+1}-r_{n}\right|,
\end{aligned}
$$

where $M_{1}$ is some real constant such that $M_{1} \geq \sup _{n \geq 1}\left\{\left\|y_{n}-x_{n}\right\|\right\}$.

On the other hand, we have

$$
\begin{aligned}
\left\|\rho_{n+1}-\rho_{n}\right\| & =\left\|P_{C}\left(I-s_{n+1} B\right) y_{n+1}-P_{C}\left(I-s_{n} B\right) y_{n}\right\| \\
& \leq\left\|\left(I-s_{n+1} B\right) y_{n+1}-\left(I-s_{n} B\right) y_{n}\right\| \\
& =\left\|\left(I-s_{n+1} B\right) y_{n+1}-\left(I-s_{n+1} B\right) y_{n}+\left(s_{n}-s_{n+1}\right) B y_{n}\right\| \\
& \leq\left\|y_{n+1}-y_{n}\right\|+\left|s_{n}-s_{n+1}\right| M_{2},
\end{aligned}
$$

where $M_{2} \geq \sup _{n \geq 1}\left\{\left\|B y_{n}\right\|\right\}$. Substituting (2.3) into (2.4) yields

$$
\left\|\rho_{n+1}-\rho_{n}\right\| \leq\left\|x_{n+1}-x_{n}\right\|+M_{3}\left(\left|r_{n+1}-r_{n}\right|+\left|s_{n}-s_{n+1}\right|\right),
$$


where $M_{3}=\max \left\{M_{1}, M_{2}\right\}$. Notice that

$$
\begin{aligned}
\left\|\zeta_{n}-\zeta_{n+1}\right\|= & \|\left(I-\beta_{n+1} A\right)\left(W_{n+1} \rho_{n+1}-W_{n} \rho_{n}\right)-\left(\beta_{n+1}-\beta_{n}\right) A W_{n} \rho_{n} \\
& +\gamma\left[\beta_{n+1}\left(f\left(y_{n+1}\right)-f\left(z_{n}\right)\right)+f\left(y_{n}\right)\left(\beta_{n+1}-\beta_{n}\right)\right] \| \\
\leq & \left(1-\beta_{n+1} \bar{\gamma}\right)\left(\left\|\rho_{n+1}-\rho_{n}\right\|+\left\|W_{n+1} \rho_{n}-W_{n} \rho_{n}\right\|\right) \\
& +\left|\beta_{n+1}-\beta_{n}\right|\left\|A W_{n} \rho_{n}\right\|+\gamma\left(\beta_{n+1} \kappa\left\|y_{n+1}-y_{n}\right\|+\left|\beta_{n+1}-\beta_{n}\right|\left\|f\left(y_{n}\right)\right\|\right)
\end{aligned}
$$

Since $T_{i}$ and $U_{n, i}$ are nonexpansive, we see from (1.3) that

$$
\begin{aligned}
\left\|W_{n+1} \rho_{n}-W_{n} \rho_{n}\right\| & =\left\|\gamma_{1} T_{1} U_{n+1,2} \rho_{n}-\gamma_{1} T_{1} U_{n, 2} \rho_{n}\right\| \\
& \leq \gamma_{1}\left\|U_{n+1,2} \rho_{n}-U_{n, 2} \rho_{n}\right\| \\
& =\gamma_{1}\left\|\gamma_{2} T_{2} U_{u+1,3} \rho_{n}-\gamma_{2} T_{2} U_{n, 3} \rho_{n}\right\| \\
& \leq \gamma_{1} \gamma_{2}\left\|U_{u+1,3} \rho_{n}-U_{n, 3} \rho_{n}\right\| \\
& \leq \cdots \\
& \leq \gamma_{1} \gamma_{2} \cdots \gamma_{n}\left\|U_{n+1, n+1} \rho_{n}-U_{n, n+1} \rho_{n}\right\| \\
& \leq M_{4} \prod_{i=1}^{n} \gamma_{i},
\end{aligned}
$$

where $M_{4}$ is a constant such that $M_{4} \geq \sup _{n \geq 1}\left\{\left\|U_{n+1, n+1} \rho_{n}-U_{n, n+1} \rho_{n}\right\|\right\}$. Substituting (2.3), (2.5) and (2.7) into (2.6) yields

$$
\left\|\zeta_{n}-\zeta_{n+1}\right\| \leq\left\|x_{n+1}-x_{n}\right\|+M_{5}\left(\prod_{i=1}^{n} \gamma_{i}+\left|r_{n+1}-r_{n}\right|+\left|s_{n}-s_{n+1}\right|+\left|\beta_{n}-\beta_{n+1}\right|\right)
$$

where $M_{5}$ is a constant such that

$$
M_{5}=\max \left\{M_{3}+\bar{\gamma} \frac{M_{1}}{m}, M_{4}, \sup _{n \geq 1}\left\{\left\|A W_{n} \rho_{n}\right\|+\gamma\left\|f\left(y_{n}\right)\right\|\right\}\right\} .
$$

It follows from the restrictions (b) and (c) that

$$
\limsup _{n \rightarrow \infty}\left(\left\|\zeta_{n}-\zeta_{n+1}\right\|-\left\|x_{n+1}-x_{n}\right\|\right) \leq 0
$$

By virtue of Lemma 1.8, we obtain that

$$
\lim _{n \rightarrow \infty}\left\|\zeta_{n}-x_{n}\right\|=0
$$

On the other hand, we have

$$
\left\|x_{n+1}-x_{n}\right\|=\left(1-\alpha_{n}\right)\left\|x_{n}-\zeta_{n}\right\| .
$$

This implies from (2.8) that

$$
\lim _{n \rightarrow \infty}\left\|x_{n+1}-x_{n}\right\|=0 .
$$

This completes the proof of Step 2 . 
Step 3. Show that $\lim _{n \rightarrow \infty}\left\|y_{n}-W y_{n}\right\|=0$.

Notice that $\zeta_{n}=\beta_{n} \gamma f\left(z_{n}\right)+\left(I-\beta_{n} A\right) W_{n} \rho_{n}$. It follows that

$$
\left\|\zeta_{n}-W_{n} \rho_{n}\right\|=\beta_{n}\left\|\gamma f\left(y_{n}\right)-A W_{n} \rho_{n}\right\|
$$

This implies from the restriction (b) that

$$
\lim _{n \rightarrow \infty}\left\|\zeta_{n}-W_{n} \rho_{n}\right\|=0
$$

For any $p \in F$, we find that

$$
\begin{aligned}
\left\|y_{n}-p\right\|^{2} & =\left\|T_{r_{n}} x_{n}-T_{r_{n}} p\right\|^{2} \\
& \leq\left\langle T_{r_{n}} x_{n}-T_{r_{n}} p, x_{n}-p\right\rangle \\
& =\left\langle y_{n}-p, x_{n}-p\right\rangle \\
& =1 / 2\left(\left\|y_{n}-p\right\|^{2}+\left\|x_{n}-p\right\|^{2}-\left\|x_{n}-y_{n}\right\|^{2}\right) .
\end{aligned}
$$

That is,

$$
\left\|y_{n}-p\right\|^{2} \leq\left\|x_{n}-p\right\|^{2}-\left\|x_{n}-y_{n}\right\|^{2} .
$$

This in turn implies that

$$
\begin{aligned}
&\left\|x_{n+1}-p\right\|^{2} \\
&=\left\|\alpha_{n}\left(x_{n}-p\right)+\left(1-\alpha_{n}\right)\left(\zeta_{n}-p\right)\right\|^{2} \\
& \leq \alpha_{n}\left\|x_{n}-p\right\|^{2}+\left(1-\alpha_{n}\right)\left\|\zeta_{n}-p\right\|^{2} \\
&=\alpha_{n}\left\|x_{n}-p\right\|^{2}+\left(1-\alpha_{n}\right)\left\|\beta_{n}\left(\gamma f\left(y_{n}\right)-A p\right)+\left(I-\beta_{n} A\right)\left(W_{n} \rho_{n}-p\right)\right\|^{2} \\
& \leq \alpha_{n}\left\|x_{n}-p\right\|^{2}+\left(1-\alpha_{n}\right)\left(\beta_{n}\left\|\gamma f\left(y_{n}\right)-A p\right\|+\left(1-\beta_{n} \bar{\gamma}\right)\left\|\rho_{n}-p\right\|\right)^{2} \\
& \leq \alpha_{n}\left\|x_{n}-p\right\|^{2}+\left(1-\alpha_{n}\right) \beta_{n}\left\|\gamma f\left(y_{n}\right)-A p\right\|^{2}+\left(1-\alpha_{n}\right)\left(1-\beta_{n} \bar{\gamma}\right)\left\|\rho_{n}-p\right\|^{2} \\
&+2\left(1-\alpha_{n}\right) \beta_{n}\left\|\gamma f\left(y_{n}\right)-A p\right\|\left\|\rho_{n}-p\right\| \\
& \leq \alpha_{n}\left\|x_{n}-p\right\|^{2}+\left(1-\alpha_{n}\right) \beta_{n}\left\|\gamma f\left(y_{n}\right)-A p\right\|^{2}+\left(1-\alpha_{n}\right)\left(1-\beta_{n} \bar{\gamma}\right)\left\|y_{n}-p\right\|^{2} \\
&+2\left(1-\alpha_{n}\right) \beta_{n}\left\|\gamma f\left(y_{n}\right)-A p\right\|\left\|\rho_{n}-p\right\| \\
& \leq \alpha_{n}\left\|x_{n}-p\right\|^{2}+\left(1-\alpha_{n}\right) \beta_{n}\left\|\gamma f\left(y_{n}\right)-A p\right\|^{2}+\left(1-\alpha_{n}\right)\left\|x_{n}-p\right\|^{2} \\
& \quad-\left(1-\alpha_{n}\right)\left(1-\beta_{n} \bar{\gamma}\right)\left\|x_{n}-y_{n}\right\|^{2}+2\left(1-\alpha_{n}\right) \beta_{n}\left\|\gamma f\left(y_{n}\right)-A p\right\|\left\|\rho_{n}-p\right\|,
\end{aligned}
$$

from which it follows that

$$
\begin{aligned}
& \left(1-\alpha_{n}\right)\left(1-\beta_{n} \bar{\gamma}\right)\left\|x_{n}-y_{n}\right\|^{2} \\
& \leq \quad\left\|x_{n}-p\right\|^{2}-\left\|x_{n+1}-p\right\|^{2}+\beta_{n}\left\|\gamma f\left(y_{n}\right)-A p\right\|^{2} \\
& \quad+2 \beta_{n}\left\|\gamma f\left(y_{n}\right)-A p\right\|\left\|\rho_{n}-p\right\|
\end{aligned}
$$




$$
\begin{aligned}
\leq & \left(\left\|x_{n}-p\right\|-\left\|x_{n+1}-p\right\|\right)\left\|x_{n}-x_{n+1}\right\|+\beta_{n}\left\|\gamma f\left(y_{n}\right)-A p\right\|^{2} \\
& +2 \beta_{n}\left\|\gamma f\left(y_{n}\right)-A p\right\|\left\|\rho_{n}-p\right\| .
\end{aligned}
$$

It follows from the restriction (b) and (2.9) that

$$
\lim _{n \rightarrow \infty}\left\|y_{n}-x_{n}\right\|=0
$$

Notice that

$$
\begin{aligned}
\left\|\rho_{n}-p\right\|^{2} & =\left\|P_{C}\left(I-s_{n} B\right) y_{n}-P_{C}\left(I-s_{n} B\right) p\right\|^{2} \\
& \leq\left\|\left(y_{n}-p\right)-s_{n}\left(B y_{n}-B p\right)\right\|^{2} \\
& =\left\|y_{n}-p\right\|^{2}-2 s_{n}\left\langle y_{n}-p, B y_{n}-B p\right\rangle+s_{n}^{2}\left\|B y_{n}-B p\right\|^{2} \\
& \leq\left\|x_{n}-p\right\|^{2}-2 s_{n} \alpha\left\|B y_{n}-B p\right\|^{2}+s_{n}^{2}\left\|B y_{n}-B p\right\|^{2} \\
& \leq\left\|x_{n}-p\right\|^{2}-s_{n}\left(2 \alpha-s_{n}\right)\left\|B y_{n}-B p\right\|^{2} .
\end{aligned}
$$

On the other hand, we have

$$
\begin{aligned}
&\left\|x_{n+1}-p\right\|^{2} \\
&=\left\|\alpha_{n}\left(x_{n}-p\right)+\left(1-\alpha_{n}\right)\left(\zeta_{n}-p\right)\right\|^{2} \\
& \leq \alpha_{n}\left\|x_{n}-p\right\|^{2}+\left(1-\alpha_{n}\right)\left\|\zeta_{n}-p\right\|^{2} \\
&= \alpha_{n}\left\|x_{n}-p\right\|^{2}+\left(1-\alpha_{n}\right)\left\|\beta_{n}\left(\gamma f\left(y_{n}\right)-A p\right)+\left(I-\beta_{n} A\right)\left(W_{n} \rho_{n}-p\right)\right\|^{2} \\
& \leq \alpha_{n}\left\|x_{n}-p\right\|^{2}+\left(1-\alpha_{n}\right)\left(\beta_{n}\left\|\gamma f\left(y_{n}\right)-A p\right\|+\left\|I-\beta_{n} A\right\|\left\|W_{n} \rho_{n}-p\right\|\right)^{2} \\
& \leq \alpha_{n}\left\|x_{n}-p\right\|^{2}+\left(1-\alpha_{n}\right)\left(\beta_{n}\left\|\gamma f\left(y_{n}\right)-A p\right\|+\left(1-\beta_{n} \bar{\gamma}\right)\left\|\rho_{n}-p\right\|\right)^{2} \\
& \leq \alpha_{n}\left\|x_{n}-p\right\|^{2}+\left(1-\alpha_{n}\right) \beta_{n}\left\|\gamma f\left(y_{n}\right)-A p\right\|^{2}+\left(1-\alpha_{n}\right)\left\|\rho_{n}-p\right\|^{2} \\
&+2\left(1-\alpha_{n}\right) \beta_{n}\left\|\gamma f\left(y_{n}\right)-A p\right\|\left\|\rho_{n}-p\right\| .
\end{aligned}
$$

Substituting (2.12) into (2.13), we find that

$$
\begin{aligned}
\left\|x_{n+1}-p\right\|^{2} \leq & \left\|x_{n}-p\right\|^{2}+\beta_{n}\left\|\gamma f\left(y_{n}\right)-A p\right\|^{2}+2 \beta_{n}\left\|\gamma f\left(y_{n}\right)-A p\right\|\left\|\rho_{n}-p\right\| \\
& -\left(1-\alpha_{n}\right) s_{n}\left(2 \alpha-s_{n}\right)\left\|B y_{n}-B p\right\|^{2} .
\end{aligned}
$$

This in turn implies that

$$
\begin{aligned}
(1- & \left.\alpha_{n}\right) s_{n}\left(2 \alpha-s_{n}\right)\left\|B y_{n}-B p\right\|^{2} \\
\leq & \left\|x_{n}-p\right\|^{2}+\beta_{n}\left\|\gamma f\left(y_{n}\right)-A p\right\|^{2}-\left\|x_{n+1}-p\right\|^{2} \\
& +2 \beta_{n}\left\|\gamma f\left(y_{n}\right)-A p\right\|\left\|\rho_{n}-p\right\| \\
\leq & \left(\left\|x_{n}-p\right\|+\left\|x_{n+1}-p\right\|\right)\left\|x_{n}-x_{n+1}\right\|+\beta_{n}\left\|\gamma f\left(y_{n}\right)-A p\right\|^{2} \\
& +2 \beta_{n}\left\|\gamma f\left(y_{n}\right)-A p\right\|\left\|\rho_{n}-p\right\| .
\end{aligned}
$$


It follows from the restrictions (a), (b) and (d) that

$$
\lim _{n \rightarrow \infty}\left\|B y_{n}-B p\right\|=0
$$

On the other hand, we have

$$
\begin{aligned}
\left\|\rho_{n}-p\right\|^{2}= & \left\|P_{C}\left(I-s_{n} B\right) y_{n}-P_{C}\left(I-s_{n} B\right) p\right\|^{2} \\
\leq & \left\langle\left(I-s_{n} B\right) y_{n}-\left(I-s_{n} B\right) p, \rho_{n}-p\right\rangle \\
= & \frac{1}{2}\left(\left\|\left(I-s_{n} B\right) y_{n}-\left(I-s_{n} B\right) p\right\|^{2}+\left\|\rho_{n}-p\right\|^{2}\right. \\
& \left.-\left\|\left(I-s_{n} B\right) y_{n}-\left(I-s_{n} B\right) p-\left(\rho_{n}-p\right)\right\|^{2}\right) \\
\leq & \frac{1}{2}\left(\left\|y_{n}-p\right\|^{2}+\left\|\rho_{n}-p\right\|^{2}-\left\|\left(y_{n}-\rho_{n}\right)-s_{n}\left(B y_{n}-B p\right)\right\|^{2}\right) \\
= & \frac{1}{2}\left(\left\|x_{n}-p\right\|^{2}+\left\|\rho_{n}-p\right\|^{2}-\left\|y_{n}-\rho_{n}\right\|^{2}-s_{n}^{2}\left\|B y_{n}-B p\right\|^{2}\right. \\
& \left.+2 s_{n}\left\|y_{n}-\rho_{n}\right\| B y_{n}-B p \|\right),
\end{aligned}
$$

which yields

$$
\left\|\rho_{n}-p\right\|^{2} \leq\left\|x_{n}-p\right\|^{2}-\left\|y_{n}-\rho_{n}\right\|^{2}+2 s_{n}\left\|y_{n}-\rho_{n}\right\|\left\|B y_{n}-B p\right\| .
$$

Substituting (2.15) into (2.13) yields

$$
\begin{aligned}
\left\|x_{n+1}-p\right\|^{2} \leq & \left\|x_{n}-p\right\|^{2}+\beta_{n}\left\|\gamma f\left(y_{n}\right)-A p\right\|^{2}-\left(1-\alpha_{n}\right)\left\|y_{n}-\rho_{n}\right\|^{2} \\
& +2 s_{n}\left\|y_{n}-\rho_{n}\right\|\left\|B y_{n}-B p\right\|+2 \beta_{n}\left\|\gamma f\left(y_{n}\right)-A p\right\|\left\|\rho_{n}-p\right\| .
\end{aligned}
$$

It follows that

$$
\begin{aligned}
\left(1-\alpha_{n}\right)\left\|y_{n}-\rho_{n}\right\|^{2} \leq & \left\|x_{n}-p\right\|^{2}-\left\|x_{n+1}-p\right\|^{2}+\beta_{n}\left\|\gamma f\left(y_{n}\right)-A p\right\|^{2} \\
& +2 s_{n}\left\|y_{n}-\rho_{n}\right\|\left\|B y_{n}-B p\right\|+2 \beta_{n}\left\|\gamma f\left(y_{n}\right)-A p\right\|\left\|\rho_{n}-p\right\| \\
\leq & \left(\left\|x_{n}-p\right\|+\left\|x_{n+1}-p\right\|\right)\left\|x_{n}-x_{n+1}\right\|+\beta_{n}\left\|\gamma f\left(y_{n}\right)-A p\right\|^{2} \\
& +2 s_{n}\left\|y_{n}-\rho_{n}\right\|\left\|B y_{n}-B p\right\|+2 \beta_{n}\left\|\gamma f\left(y_{n}\right)-A p\right\|\left\|\rho_{n}-p\right\| .
\end{aligned}
$$

In view of the restrictions (a), (b) and (d), we find from (2.9) that

$$
\lim _{n \rightarrow \infty}\left\|y_{n}-\rho_{n}\right\|=0
$$

Notice that

$$
\begin{aligned}
\left\|y_{n}-W_{n} y_{n}\right\| & \leq\left\|W_{n} y_{n}-W_{n} \rho_{n}\right\|+\left\|W_{n} \rho_{n}-\zeta_{n}\right\|+\left\|\zeta_{n}-x_{n}\right\|+\left\|x_{n}-y_{n}\right\| \\
& \leq\left\|y_{n}-\rho_{n}\right\|+\left\|W_{n} \rho_{n}-\zeta_{n}\right\|+\left\|\zeta_{n}-x_{n}\right\|+\left\|x_{n}-y_{n}\right\| .
\end{aligned}
$$


In the light of (2.8), (2.10), (2.11) and (2.16), we find that $\lim _{n \rightarrow \infty}\left\|y_{n}-W_{n} y_{n}\right\|=0$. On the other hand, we have

$$
\left\|W y_{n}-y_{n}\right\| \leq\left\|W y_{n}-W_{n} y_{n}\right\|+\left\|W_{n} y_{n}-y_{n}\right\| .
$$

It follows from Lemma 1.9 that

$$
\lim _{n \rightarrow \infty}\left\|y_{n}-W y_{n}\right\|=0
$$

This completes the proof of Step 3.

Step 4. Show that $\lim _{\sup } \rightarrow \infty\left\langle\gamma f(q)-A q, x_{n}-q\right\rangle \leq 0$, where $q=P_{F}(\gamma f+(I-A))(q)$.

To see this, we choose a subsequence $\left\{x_{n_{i}}\right\}$ of $\left\{x_{n}\right\}$ such that

$$
\limsup _{n \rightarrow \infty}\left\langle\gamma f(q)-A q, x_{n}-q\right\rangle=\lim _{i \rightarrow \infty}\left\langle\gamma f(q)-A q, x_{n_{i}}-q\right\rangle .
$$

Correspondingly, there exists a subsequence $\left\{y_{n_{i}}\right\}$ of $\left\{y_{n}\right\}$. Since $\left\{y_{n_{i}}\right\}$ is bounded, there exists a subsequence $\left\{y_{n_{i}}\right\}$ of $\left\{y_{n_{i}}\right\}$ which converges weakly to $w$. Without loss of generality, we can assume that $y_{n_{i}} \rightarrow w$. Since $y_{n}=T_{r_{n}} x_{n}$, we have

$$
F\left(y_{n}, z\right)+\frac{1}{r_{n}}\left\langle z-y_{n}, y_{n}-x_{n}\right\rangle \geq 0, \quad \forall z \in C .
$$

It follows from (A2) that

$$
\left\langle z-y_{n}, \frac{y_{n}-x_{n}}{r_{n}}\right\rangle \geq F\left(z, y_{n}\right) \text {. }
$$

It follows that

$$
\left\langle z-y_{n_{i}}, \frac{y_{n_{i}}-x_{n_{i}}}{r_{n_{i}}}\right\rangle \geq F\left(z, y_{n_{i}}\right) .
$$

In view of the restriction (c), we obtain from (2.11) that

$$
\lim _{n \rightarrow \infty} \frac{y_{n}-x_{n}}{r_{n}}=0 .
$$

Since $y_{n_{i}} \rightarrow w$, we have from (A4) that $F(z, w) \leq 0$ for all $z \in C$. For $t$ with $0<t \leq 1$ and $z \in C$, let $z_{t}=t z+(1-t) w$. Since $z \in C$ and $w \in C$, we have $z_{t} \in C$ and hence $F\left(z_{t}, w\right) \leq 0$. So, from (A1) and (A4), we have

$$
0=F\left(z_{t}, z_{t}\right) \leq t F\left(z_{t}, z\right)+(1-t) F\left(z_{t}, w\right) \leq t F\left(z_{t}, z\right) .
$$

That is, $F\left(z_{t}, z\right) \geq 0$. It follows from (A3) that $F(w, z) \geq 0$ for all $z \in C$ and hence $w \in E P(F)$. On the other hand, we see that $w \in F(W)=\bigcap_{i=1}^{\infty} F\left(T_{i}\right)$. If $w \neq W w$, then we have the following. Since Hilbert spaces are Opial's spaces, we find from (2.17) that

$$
\begin{aligned}
\liminf _{i \rightarrow \infty}\left\|y_{n_{i}}-w\right\| & <\liminf _{i \rightarrow \infty}\left\|y_{n_{i}}-W w\right\| \\
& =\liminf _{i \rightarrow \infty}\left\|y_{n_{i}}-W y_{n_{i}}+W y_{n_{i}}-W w\right\|
\end{aligned}
$$




$$
\begin{aligned}
& \leq \liminf _{i \rightarrow \infty}\left\|W y_{n_{i}}-W w\right\| \\
& \leq \liminf _{i \rightarrow \infty}\left\|y_{n_{i}}-w\right\|
\end{aligned}
$$

which derives a contradiction. Thus, we have $w \in F(W)$. Next, let us first show that $w \in$ $V I(C, B)$. Put

$$
S \xi= \begin{cases}B \xi+N_{C} \xi, & \xi \in C, \\ \emptyset, & \xi \bar{\in} C .\end{cases}
$$

Since $B$ is monotone, we see that $S$ is maximal monotone. Let $\left(\xi, \xi^{\prime}\right) \in \operatorname{Graph}(S)$. Since $\xi^{\prime}-B \xi \in N_{C} \xi$ and $\rho_{n} \in C$, we have

$$
\left\langle\xi-\rho_{n}, \xi^{\prime}-B \xi\right\rangle \geq 0
$$

On the other hand, we have from $\rho_{n}=P_{C}\left(I-s_{n} B\right) y_{n}$ that

$$
\left\langle\xi-\rho_{n}, \rho_{n}-\left(I-s_{n} B\right) y_{n}\right\rangle \geq 0
$$

That is,

$$
\left\langle\xi-\rho_{n}, \frac{\rho_{n}-y_{n}}{s_{n}}+B y_{n}\right\rangle \geq 0 .
$$

It follows from the above that

$$
\begin{aligned}
\left\langle\xi-\rho_{n_{i}}, \xi^{\prime}\right\rangle \geq & \left\langle\xi-\rho_{n_{i}}, B \xi\right\rangle \\
\geq & \left\langle\xi-\rho_{n_{i}}, B \xi-\frac{\rho_{n_{i}}-y_{n_{i}}}{s_{n_{i}}}-B y_{n_{i}}\right\rangle \\
= & \left\langle\xi-\rho_{n_{i}}, B \xi-B \rho_{n_{i}}\right\rangle+\left\langle\xi-\rho_{n_{i}}, B \rho_{n_{i}}-B y_{n_{i}}\right\rangle \\
& -\left\langle\xi-\rho_{n_{i}}, \frac{\rho_{n_{i}}-y_{n_{i}}}{s_{n_{i}}}\right\rangle \\
\geq & \left\langle\xi-\rho_{n_{i}}, B \rho_{n_{i}}-B y_{n_{i}}\right\rangle-\left\langle\xi-\rho_{n_{i}}, \frac{\rho_{n_{i}}-y_{n_{i}}}{s_{n_{i}}}\right\rangle,
\end{aligned}
$$

which implies from (2.16) that $\left\langle\xi-w, \xi^{\prime}\right\rangle \geq 0$. We have $w \in S^{-1} 0$ and hence $w \in V I(C, B)$. This completes the proof $w \in F$. On the other hand, we find from (2.18) that

$$
\begin{aligned}
\limsup _{n \rightarrow \infty}\left\langle\gamma f(q)-A q, x_{n}-q\right\rangle & =\lim _{n \rightarrow \infty}\left\langle\gamma f(q)-A q, x_{n_{i}}-q\right\rangle \\
& =\langle\gamma f(q)-A q, w-q\rangle \leq 0 .
\end{aligned}
$$

This completes the proof of Step 4.

Step 5. Show $\lim _{n \rightarrow \infty}\left\|x_{n}-q\right\|=0$. 
It follows from Lemma 1.3 that

$$
\begin{aligned}
\left\|\zeta_{n}-q\right\|^{2}= & \left\|\left(I-\beta_{n} A\right)\left(W_{n} \rho_{n}-q\right)+\beta_{n}\left(\gamma f\left(y_{n}\right)-A q\right)\right\|^{2} \\
\leq & \left\|\left(I-\beta_{n} A\right)\left(W_{n} \rho_{n}-q\right)\right\|^{2}+2 \beta_{n}\left\langle\gamma f\left(z_{n}\right)-A q, \zeta_{n}-q\right\rangle \\
\leq & \left(1-\beta_{n} \bar{\gamma}\right)^{2}\left\|\rho_{n}-q\right\|^{2}+2 \beta_{n}\left\langle\gamma f\left(y_{n}\right)-A q, \zeta_{n}-q\right\rangle \\
\leq & \left(1-\beta_{n} \bar{\gamma}\right)^{2}\left\|y_{n}-q\right\|^{2}+2 \beta_{n} \gamma\left\langle f\left(y_{n}\right)-f(q), \zeta_{n}-q\right\rangle \\
& +2 \beta_{n}\left\langle\gamma f(q)-A q, \zeta_{n}-q\right\rangle \\
\leq & \left(1-\beta_{n} \bar{\gamma}\right)^{2}\left\|x_{n}-q\right\|^{2}+2 \beta_{n} \gamma \kappa\left\|y_{n}-q\right\|\left\|\zeta_{n}-q\right\| \\
& +2 \beta_{n}\left\langle\gamma f(q)-A q, \zeta_{n}-q\right\rangle \\
\leq & \left(1-\beta_{n} \bar{\gamma}\right)^{2}\left\|x_{n}-q\right\|^{2}+\beta_{n} \gamma \kappa\left(\left\|y_{n}-q\right\|^{2}+\left\|\zeta_{n}-q\right\|^{2}\right) \\
& +2 \beta_{n}\left\langle\gamma f(q)-A q, \zeta_{n}-q\right\rangle \\
\leq & \left(1-\beta_{n} \bar{\gamma}\right)^{2}\left\|x_{n}-q\right\|^{2}+\beta_{n} \gamma \kappa\left(\left\|x_{n}-q\right\|^{2}+\left\|\zeta_{n}-q\right\|^{2}\right) \\
& +2 \beta_{n}\left\langle\gamma f(q)-A q, \zeta_{n}-q\right\rangle,
\end{aligned}
$$

which implies that

$$
\begin{aligned}
\left\|\zeta_{n}-q\right\|^{2} \leq & \frac{\left(1-\beta_{n} \bar{\gamma}\right)^{2}+\beta_{n} \gamma \kappa}{1-\beta_{n} \gamma \alpha}\left\|x_{n}-q\right\|^{2}+\frac{2 \beta_{n}}{1-\beta_{n} \gamma \kappa}\left\langle\gamma f(q)-A q, \zeta_{n}-q\right\rangle \\
= & \frac{\left(1-2 \beta_{n} \bar{\gamma}+\beta_{n} \kappa \gamma\right)}{1-\beta_{n} \gamma \kappa}\left\|x_{n}-q\right\|^{2}+\frac{\beta_{n}^{2} \bar{\gamma}^{2}}{1-\beta_{n} \gamma \kappa}\left\|x_{n}-q\right\|^{2} \\
& +\frac{2 \beta_{n}}{1-\beta_{n} \gamma \kappa}\left\langle\gamma f(q)-A q, \zeta_{n}-q\right\rangle \\
\leq & \left(1-\frac{2 \beta_{n}(\bar{\gamma}-\kappa \gamma)}{1-\beta_{n} \gamma \kappa}\right)\left\|x_{n}-q\right\|^{2} \\
& +\frac{2 \beta_{n}(\bar{\gamma}-\kappa \gamma)}{1-\beta_{n} \gamma \kappa}\left(\frac{1}{\bar{\gamma}-\kappa \gamma}\left\langle\gamma f(q)-A q, \zeta_{n}-q\right\rangle+\frac{\beta_{n} \bar{\gamma}^{2}}{2(\bar{\gamma}-\kappa \gamma)} M_{6}\right),
\end{aligned}
$$

where $M_{6}$ is a constant such that $M_{6} \geq \sup _{n \geq 1}\left\{\left\|x_{n}-q\right\|^{2}\right\}$. On the other hand, we have

$$
\left\|x_{n+1}-p\right\|^{2} \leq \alpha_{n}\left\|x_{n}-p\right\|^{2}+\left(1-\alpha_{n}\right)\left\|\zeta_{n}-p\right\|^{2}
$$

Substituting (2.20) into (2.21) yields

$$
\begin{aligned}
\left\|x_{n+1}-p\right\|^{2} \leq & {\left[1-\left(1-\alpha_{n}\right) \frac{2 \beta_{n}(\bar{\gamma}-\kappa \gamma)}{1-\beta_{n} \gamma \kappa}\right]\left\|x_{n}-q\right\|^{2} } \\
& +\left(1-\alpha_{n}\right) \frac{2 \beta_{n}(\bar{\gamma}-\kappa \gamma)}{1-\beta_{n} \gamma \alpha} \\
& \times\left(\frac{1}{\bar{\gamma}-\kappa \gamma}\left\langle\gamma f(q)-A q, \zeta_{n}-q\right\rangle+\frac{\beta_{n} \bar{\gamma}^{2}}{2(\bar{\gamma}-\kappa \gamma)} M_{6}\right) .
\end{aligned}
$$


Let $\lambda_{n}=\left(1-\alpha_{n}\right) \frac{2 \beta_{n}(\bar{\gamma}-\kappa \gamma)}{1-\beta_{n} \kappa \gamma}$ and

$$
\theta_{n}=\frac{1}{\bar{\gamma}-\kappa \gamma}\left\langle\gamma f(q)-A q, \zeta_{n}-q\right\rangle+\frac{\beta_{n} \bar{\gamma}^{2}}{2(\bar{\gamma}-\kappa \gamma)} M_{6}
$$

This implies that

$$
\left\|x_{n+1}-q\right\|^{2} \leq\left(1-\lambda_{n}\right)\left\|x_{n}-q\right\|^{2}+\lambda_{n} t_{n}
$$

In view of the restriction (b), we find from (2.8) and (2.11) that

$$
\lim _{n \rightarrow \infty} \lambda_{n}=0, \quad \sum_{n=1}^{\infty} \lambda_{n}=\infty \quad \text { and } \quad \limsup _{n \rightarrow \infty} \theta_{n} \leq 0 .
$$

We can easily draw the desired conclusion with the aid of Lemma 1.4. This completes the proof of Step 5. The proof is completed.

From Theorem 2.1, we have the following results.

Corollary 2.2 Let $C$ be a nonempty closed convex subset of a Hilbert space H. Let $\left\{T_{n}\right\}_{n=1}^{\infty}$ be an infinite family of nonexpansive mappings of $C$ into $C$. Let $B: C \rightarrow H$ be an $\alpha$-inversestrongly monotone mapping. Let $A$ be a strongly positive linear bounded self-adjoint operator on $H$ with the coefficient $\bar{\gamma}>0$. Assume that $0<\gamma<\bar{\gamma} / \kappa$ and $F:=\bigcap_{i=1}^{\infty} F\left(T_{i}\right) \cap V I(C, B) \neq$ $\emptyset$. Let $f: C \rightarrow H$ be a $\kappa$-contraction. Let $\left\{x_{n}\right\}$ be a sequence generated in the following iterative process:

$$
\left\{\begin{array}{l}
x_{1} \in H, \\
x_{n+1}=\alpha_{n} x_{n}+\left(1-\alpha_{n}\right) \beta_{n} \gamma f\left(y_{n}\right)+\left(1-\alpha_{n}\right)\left(I-\beta_{n} A\right) W_{n} P_{C}\left(I-s_{n} B\right) P_{C} x_{n}, \quad n \geq 1,
\end{array}\right.
$$

where $W_{n}$ is generated in (1.3), $\left\{\alpha_{n}\right\},\left\{\beta_{n}\right\}$ are real number sequences in $(0,1),\left\{r_{n}\right\}$ and $\left\{s_{n}\right\}$ are positive real number sequences. Assume that the following restrictions are satisfied:

(a) $0<\liminf _{n \rightarrow \infty} \alpha_{n} \leq \limsup _{n \rightarrow \infty} \alpha_{n}<1$;

(b) $\lim _{n \rightarrow \infty} \beta_{n}=0, \sum_{n=1}^{\infty} \beta_{n}=\infty$;

(c) $\lim _{n \rightarrow \infty}\left|s_{n+1}-s_{n}\right|=0$;

(d) $\left\{s_{n}\right\} \subset\left[s, s^{\prime}\right]$ for some $s, s^{\prime}$ with $0<s<s^{\prime}<2 \alpha$.

Then $\left\{x_{n}\right\}$ converges strongly to $q \in F$, where $q=P_{F}(\gamma f+(I-A))(q)$, which solves the following variational inequality:

$$
\langle\gamma f(q)-A q, p-q\rangle \leq 0, \quad \forall p \in F
$$

Proof Putting $F(x, y)=0$ and $r_{n}=1$, we can immediately draw the desired conclusion from Theorem 2.1.

Corollary 2.3 Let $C$ be a nonempty closed convex subset of a Hilbert space H. Let $F$ be a bifunction from $C \times C$ to $\mathbb{R}$, which satisfies (A1)-(A4). Let $B: C \rightarrow H$ be an $\alpha$-inverse-strongly monotone mapping. Let $A$ be a strongly positive linear bounded self-adjoint operator on $H$ with the coefficient $\bar{\gamma}>0$. Assume that $0<\gamma<\bar{\gamma} / \kappa$ and $F:=E P(F) \cap V I(C, B) \neq \emptyset$. Let 
$f: C \rightarrow H$ be a $\kappa$-contraction. Let $\left\{x_{n}\right\}$ be a sequence generated in the following iterative process:

$$
\left\{\begin{array}{l}
x_{1} \in H, \\
F\left(y_{n}, z\right)+\frac{1}{r_{n}}\left\langle z-y_{n}, y_{n}-x_{n}\right\rangle \geq 0, \quad \forall z \in C, \\
x_{n+1}=\alpha_{n} x_{n}+\left(1-\alpha_{n}\right) \beta_{n} \gamma u+\left(1-\alpha_{n}\right)\left(I-\beta_{n} A\right) P_{C}\left(I-s_{n} B\right) y_{n}, \quad n \geq 1,
\end{array}\right.
$$

where $u$ is a fixed element in $C,\left\{\alpha_{n}\right\},\left\{\beta_{n}\right\}$ are real number sequences in $(0,1),\left\{r_{n}\right\}$ and $\left\{s_{n}\right\}$ are positive real number sequences. Assume that the following restrictions are satisfied:

(a) $0<\liminf _{n \rightarrow \infty} \alpha_{n} \leq \limsup _{n \rightarrow \infty} \alpha_{n}<1$;

(b) $\lim _{n \rightarrow \infty} \beta_{n}=0, \sum_{n=1}^{\infty} \beta_{n}=\infty$;

(c) $\lim _{n \rightarrow \infty}\left|r_{n+1}-r_{n}\right|=0, \lim _{n \rightarrow \infty}\left|s_{n+1}-s_{n}\right|=0$;

(d) $\liminf _{n \rightarrow \infty} r_{n}>0,\left\{s_{n}\right\} \subset\left[s, s^{\prime}\right]$ for some $s, s^{\prime}$ with $0<s<s^{\prime}<2 \alpha$.

Then, $\left\{x_{n}\right\}$ converges strongly to $q \in F$, where $q=P_{F}(\gamma u+(q-A q))$, which solves the following variational inequality:

$$
\langle\gamma u-A q, p-q\rangle \leq 0, \quad \forall p \in F
$$

Proof Putting $T_{i}=I$, where $I$ is the identity mapping and $f(x)=u$, for all $x \in C$, we can immediately draw the desired conclusion from Theorem 2.1.

Corollary 2.4 Let $C$ be a nonempty closed convex subset of a Hilbert space H. Let $F$ be a bifunction from $C \times C$ to $\mathbb{R}$ which satisfies (A1)-(A4). Let $\left\{T_{n}\right\}_{n=1}^{\infty}$ be an infinite family of nonexpansive mappings of $C$ into $C$. Let $B: C \rightarrow H$ be an $\alpha$-inverse-strongly monotone mapping. Assume that $F:=\bigcap_{i=1}^{\infty} F\left(T_{i}\right) \cap E P(F) \cap V I(C, B) \neq \emptyset$. Let $f: C \rightarrow H$ be a $\kappa$-contraction. Let $\left\{x_{n}\right\}$ be a sequence generated in the following iterative process:

$$
\left\{\begin{array}{l}
x_{1} \in H, \\
F\left(y_{n}, z\right)+\frac{1}{r_{n}}\left\langle z-y_{n}, y_{n}-x_{n}\right\rangle \geq 0, \quad \forall z \in C, \\
x_{n+1}=\alpha_{n} x_{n}+\left(1-\alpha_{n}\right) \beta_{n} f\left(y_{n}\right)+\left(1-\alpha_{n}\right)\left(1-\beta_{n}\right) W_{n} P_{C}\left(I-s_{n} B\right) y_{n}, \quad n \geq 1,
\end{array}\right.
$$

where $W_{n}$ is generated in (1.3), $\left\{\alpha_{n}\right\},\left\{\beta_{n}\right\}$ are real number sequences in $(0,1),\left\{r_{n}\right\}$ and $\left\{s_{n}\right\}$ are positive real number sequences. Assume that the following restrictions are satisfied:

(a) $0<\liminf _{n \rightarrow \infty} \alpha_{n} \leq \limsup _{n \rightarrow \infty} \alpha_{n}<1$;

(b) $\lim _{n \rightarrow \infty} \beta_{n}=0, \sum_{n=1}^{\infty} \beta_{n}=\infty$;

(c) $\lim _{n \rightarrow \infty}\left|r_{n+1}-r_{n}\right|=0, \lim _{n \rightarrow \infty}\left|s_{n+1}-s_{n}\right|=0$;

(d) $\liminf _{n \rightarrow \infty} r_{n}>0,\left\{s_{n}\right\} \subset\left[s, s^{\prime}\right]$ for some $s, s^{\prime}$ with $0<s<s^{\prime}<2 \alpha$.

Then $\left\{x_{n}\right\}$ converges strongly to $q \in F$, where $q=P_{F} f(q)$, which solves the following variational inequality:

$$
\langle f(q)-q, p-q\rangle \leq 0, \quad \forall p \in F
$$

Proof Putting $A=I$, where $I$ is the identity mapping and $\gamma=1$, we can immediately draw the desired conclusion from Theorem 2.1. 


\section{Competing interests}

The author declares that they have no competing interests.

\section{Received: 13 September 2012 Accepted: 16 December 2012 Published: 15 January 2013}

\section{References}

1. Rockafellar, RT: On the maximality of sums of nonlinear monotone operators. Trans. Am. Math. Soc. 149, 75-88 (1970)

2. Su, Y, Shang, M, Qin, X: An iterative method of solution for equilibrium and optimization problems. Nonlinear Anal. 69, 2709-2719 (2008)

3. Zegeye, H, Shahzad, N, Alghamdi, M: Strong convergence theorems for a common point of solution of variational inequality, solutions of equilibrium and fixed point problems. Fixed Point Theory Appl. 2012, 119 (2012)

4. Kang, SM, Cho, SY, Liu, Z: Convergence of iterative sequences for generalized equilibrium problems involving inverse-strongly monotone mappings. J. Inequal. Appl. 2010, 827082 (2010)

5. Cho, SY, Qin, X, Kang, SM: Hybrid projection algorithms for treating common fixed points of a family of demicontinuous pseudocontractions. Appl. Math. Lett. 25, 854-857 (2012)

6. Yang, S, Li, W: Iterative solutions of a system of equilibrium problems in Hilbert spaces. Adv. Fixed Point Theory 1 , 15-26 (2011)

7. Cho, SY, Kang, SM: Approximation of common solutions of variational inequalities via strict pseudocontractions. Acta Math. Sci. 32, 1607-1618 (2012)

8. Cho, SY, Kang, SM: Approximation of fixed points of pseudocontraction semigroups based on a viscosity iterative process. Appl. Math. Lett. 24, 224-228 (2011)

9. Kim, JK: Strong convergence theorems by hybrid projection methods for equilibrium problems and fixed point problems of the asymptotically quasi- $\phi$-nonexpansive mappings. Fixed Point Theory Appl. 2011, 10 (2011)

10. Zegeye, $\mathrm{H}$, Shahzad, N: Strong convergence theorem for a common point of solution of variational inequality and fixed point problem. Adv. Fixed Point Theory 2, 374-397 (2012)

11. Ye, J, Huang, J: Strong convergence theorems for fixed point problems and generalized equilibrium problems of three relatively quasi-nonexpansive mappings in Banach spaces. J. Math. Comput. Sci. 1, 1-18 (2011)

12. Qin, X, Cho, YJ, Kang, SM: Convergence theorems of common elements for equilibrium problems and fixed point problems in Banach spaces. J. Comput. Appl. Math. 225, 20-30 (2009)

13. Qin, X, Cho, SY, Kang, SM: Strong convergence of shrinking projection methods for quasi- $\phi$-nonexpansive mappings and equilibrium problems. J. Comput. Appl. Math. 234, 750-760 (2010)

14. LV, S, Wu, C: Convergence of iterative algorithms for a generalized variational inequality and a nonexpansive mapping. Eng. Math. Lett. 1, 44-57 (2012)

15. Blum, E, Oettli, W: From optimization and variational inequalities to equilibrium problems. Math. Stud. 63, 123-145 (1994)

16. Combettes, PL, Hirstoaga, SA: Equilibrium programming in Hilbert spaces. J. Nonlinear Convex Anal. 6, 117-136 (2005)

17. Takahashi, S, Takahashi, W: Viscosity approximation methods for equilibrium problems and fixed point problems in Hilbert spaces. J. Math. Anal. Appl. 331, 506-515 (2007)

18. Qin, X, Chang, SS, Cho, YJ: Iterative methods for generalized equilibrium problems and fixed point problems with applications. Nonlinear Anal. 11, 2963-2972 (2010)

19. Plubtieng, S, Punpaeng, R: A general iterative method for equilibrium problems and fixed point problems in Hilbert spaces. J. Math. Anal. Appl. 336, 455-469 (2007)

20. Shimoji, K, Takahashi, W: Strong convergence to common fixed points of infinite nonexpansive mappings and applications. Taiwan. J. Math. 5, 387-404 (2001)

21. Xu, HK: An iterative approach to quadratic optimization. J. Optim. Theory Appl. 116, 659-678 (2003)

22. Marino, G, Xu, HK: A general iterative method for nonexpansive mappings in Hilbert spaces. J. Math. Anal. Appl. 318, 43-52 (2006)

23. Blum, E, Oettli, W: From optimization and variational inequalities to equilibrium problems. Math. Stud. 63, 123-145 (1994)

24. Combettes, PL, Hirstoaga, SA: Equilibrium programming in Hilbert spaces. J. Nonlinear Convex Anal. 6, 117-136 (2005)

25. Suzuki, T: Strong convergence of Krasnoselskii and Mann's type sequences for one-parameter nonexpansive semigroups without Bochner integrals. J. Math. Anal. Appl. 305, 227-239 (2005)

26. Ceng, LC, Yao, JC: Hybrid viscosity approximation schemes for equilibrium problems and fixed point problems of infinitely many nonexpansive mappings. Appl. Math. Comput. 198, 729-741 (2008)

27. Chang, SS, Lee, HWJ, Chan, CK: A new method for solving equilibrium problem fixed point problem and variational inequality problem with application to optimization. Nonlinear Anal. 70, 3307-3319 (2009) 